\title{
Angular dihadron correlations as an interplay between elliptic and triangular flows
}

\author{
G. Eyyubova ${ }^{*}$ \\ Faculty of Nuclear Sciences and Physical Engineering, \\ Czech Technical University in Prague, CR-11519 Prague, Czech Republic \\ V.L. Korotkikh, I.P. Lokhtin, S.V. Petrushanko, and A.M. Snigirev \\ Skobeltsyn Institute of Nuclear Physics, Moscow State University, RU-119991 Moscow, Russia \\ L. Bravina and E.E. Zabrodin† \\ Department of Physics, University of Oslo, PB 1048 Blindern, N-0316 Oslo, Norway
}

\begin{abstract}
The hybrid model HYDJET++, which considers soft and hard processes, is employed for the analysis of dihadron angular correlations measured in $\mathrm{Pb}+\mathrm{Pb}$ collisions at center-of-mass energy $\sqrt{s_{\mathrm{NN}}}=2.76 \mathrm{TeV}$. The model allows the study of both individual and mutual influence of lower flow harmonics, $v_{2}$ and $v_{3}$, on higher harmonics and dihadron angular correlations. It is shown that the typical structure called a ridge in dihadron angular correlations in a broad pseudorapidity range could appear just as interplay of $v_{2}$ and $v_{3}$. Central, semi-central and semi-peripheral collisions were investigated. Comparison of model results with the experimental data on dihadron angular correlations is presented for different centralities and transverse momenta $p_{\mathrm{T}}$.
\end{abstract}

PACS numbers: 25.75.-q, 25.75.Ld, 24.10.Nz, 25.75.Bh

\section{INTRODUCTION}

The measurement of azimuthal anisotropy and angular correlations of particles is an important tool for exploring properties of matter produced in nucleus-nucleus collisions. For non-central collisions of nuclei the initial azimuthal anisotropic overlap region leads to anisotropies in final particle distribution over the azimuth $d N / d \varphi$, which is characterized by the coefficients $v_{n}$ in the Fourier decomposition

$$
\frac{d N}{d \varphi} \propto 1+2 \sum_{n=1}^{\infty} v_{n}\left(p_{\mathrm{T}}, \eta\right) \cos \left[n\left(\varphi-\Psi_{n}\right)\right]
$$

where $\Psi_{n}$ is the azimuth of the participant event plane of the $n$th harmonic, and coefficients $v_{n}$ depend on the transverse momentum $p_{\mathrm{T}}$ and pseudorapidity $\eta$. The two-particle angular correlation function, $C(\Delta \eta, \Delta \varphi)$, in the relative pseudorapidity $\Delta \eta=\eta^{\operatorname{tr}}-\eta^{\mathrm{a}}$ and the azimuth $\Delta \varphi=\varphi^{\mathrm{tr}}-\varphi^{\mathrm{a}}$ is sensitive to the collective flow of particles as well as to any other particle correlations in azimuthal angle and pseudorapidity. In the flow dominated regime the pair distribution can be expanded in a Fourier series:

$$
\frac{d N^{\text {pairs }}}{d \Delta \varphi} \propto 1+2 \sum_{n=1}^{\infty} V_{n}\left(p_{\mathrm{T}}^{\mathrm{tr}}, p_{\mathrm{T}}^{\mathrm{a}}\right) \cos (n \Delta \varphi)
$$

\footnotetext{
* Also at Skobeltsyn Institute of Nuclear Physics, Moscow State University, RU-119991 Moscow, Russia

$\dagger$ Also at Skobeltsyn Institute of Nuclear Physics, Moscow State University, RU-119991 Moscow, Russia
}

where superscript indices refer to the two particles in a pair, usually called "trigger" and "associated" ones. The study of dihadron angular correlations in heavy ion collisions has revealed the new phenomena in collision dynamics, the so-called ridge and double-hump structure [1-3. In order to explain this correlation structure many mechanisms have been proposed, such as conical emission from either Mach-cone shock waves [4, 5] or Cerenkov gluon radiation [6, large-angle gluon radiation, jets deflected by radial flow and path-length dependent energy loss (see [7] and references therein).

In Ref. 8 the authors suggested that triangular flow might play an important role in the understanding of the ridge nature. Triangular flow, as well as higher flow harmonics, should arise due to initial state fluctuations in a collision geometry. Then, experiments at the CERN Large Hadron Collider (LHC) provided us with a new set of amazing results. Particularly, the ridge structure in a two-dimensional correlation function was also observed in proton-lead [9 12] and in high multiplicity proton-proton collisions [13]. The origin of the ridgelike structure in $p p$ interactions and its similarity to that in $\mathrm{Pb}+\mathrm{Pb}$ collisions are still open questions. Triangular flow measured in $p+\mathrm{Pb}$ reactions appeared to be compatible with $v_{3}$ in lead-lead collisions provided the multiplicity of secondary hadrons was the same. Traditionally proton-nucleus collisions are considered as cold nuclear matter effects, hence, the question is, Can the azimuthal anisotropy in cold nuclear matter have the the same strength as in hot nuclear matter?

In heavy ion collisions the long-range, i.e., $|\Delta \eta|>2$, angular dihadron correlations at low and intermediate transverse momenta in (mid)central collisions were shown to be described with the sum of the Fourier harmonics $v_{2} \div v_{6}$, found from independent flow analysis [3, 14, 15]. 
This implies that the $V_{n}$ coefficients in Eq. (2) factorize into two single-particle flow coefficients

$$
\frac{d N^{\text {pairs }}}{d \Delta \varphi} \propto 1+2 \sum_{n=2}^{\infty} v_{n}\left(p_{\mathrm{T}}^{\mathrm{tr}}\right) v_{n}\left(p_{\mathrm{T}}^{\mathrm{a}}\right) \cos (n \Delta \varphi) .
$$

The factorization was found to break at higher $p_{\mathrm{T}}$ and also for the first coefficient $V_{1}$ for the entire $p_{\mathrm{T}}$ range [3, 15. Are all of the six harmonics equally important for the description of long-range correlations?

In the present paper we are going to study the role of only elliptic $v_{2}$ and triangular $v_{3}$ flows in the formation of long-range correlations. For this purpose we employ the HYDJET ++ model [16, which merges parametrized hydrodynamics with jets. In addition to hard processes, the unique feature of the model is the possibility to switch on and off the elliptic and triangular harmonics in order to investigate both their individual contributions and the result of mutual interplay to the considered phenomena. The dihadron correlation function $C(\Delta \eta, \Delta \varphi)$ in leadlead collisions at $\sqrt{s_{\mathrm{NN}}}=2.76 \mathrm{TeV}$ is investigated. The appearance of higher order harmonics $V_{n}, n>3$ in the correlation function is checked, and the obtained results are compareded against the available experimental data.

\section{HYDJET + + MODEL}

The basic features of HYDJET ++ model are described in a manual [16. The model combines two components corresponding to soft and hard processes. The parameter which regulates the contribution of each component to the total event is the minimal transverse momentum $p_{\mathrm{T}}^{\min }$ of hard scattering. The partons either produced at or quenched down to the momenta below $p_{\mathrm{T}}^{\min }$ are considered to be thermal ones. Such partons do not contribute to the hard part.

The hard part of the model is based on PYTHIA [17] and PYQUEN [18] generators, which simulate parton-parton collisions, parton radiative energy loss, and hadronization. The soft part of the model has no evolution stage from the initial state until hadronization, but rather represents a thermal hadron production already at the freeze-out hypersurface in accordance with the prescriptions of ideal hydrodynamics adapted from the event generator FAST MC [19].

Strength and direction of the elliptic flow $v_{2}$ are regulated in the HYDJET ++ by two parameters. Spatial anisotropy $\epsilon(b)$ represents the elliptic modulation of the final freeze-out hypersurface at a given impact parameter $b$, whereas momentum anisotropy $\delta(b)$ deals with the modulation of flow velocity profile. Additional triangular modulation of the freeze-out hypersurface,

$$
\begin{aligned}
R(\varphi, b) & \propto \frac{\sqrt{1-\epsilon(b)}}{\sqrt{1+\epsilon(b) \cos 2\left[\left(\varphi-\Psi_{2}\right]\right)}} \\
& \times\left\{1+\epsilon_{3}(b) \cos \left[3\left(\varphi-\Psi_{3}\right)\right]\right\},
\end{aligned}
$$

produces triangular flow $v_{3}[20,21]$. Here $\epsilon_{3}$ is the new anisotropy parameter. The reaction plane $\Psi_{2}$ is fixed to zero and the $\Psi_{3}$ plane is generated randomly on an eventby-event basis. Both planes do not depend on $p_{\mathrm{T}}$ and $\eta$. Thus, the two planes are uncorrelated in accordance with the experimental data. The recent version of HYDJET ++ is tuned to describe data on lead-lead collisions at the LHC energies 20, 21].

\section{HYDJET++ AND DIHADRON CORRELATIONS}

The two-particle correlation function is defined as the ratio of pair distribution in the same event (signal) to the combinatorial pair distribution (background), where pairs are not correlated. In experiment the background function is usually constructed with pairs from mixed events. The ATLAS and ALICE collaborations use the following definition [3, 15]:

$C(\Delta \eta, \Delta \varphi) \equiv \frac{d^{2} N^{\text {pair }}}{d \Delta \eta d \Delta \varphi}=\frac{N^{\text {mixed }}}{N^{\text {same }}} \times \frac{d^{2} N^{\text {same }} / d \Delta \eta d \Delta \varphi}{d^{2} N^{\text {mixed }} / d \Delta \eta d \Delta \varphi}$,

where $N^{\text {mixed }}$ and $N^{\text {same }}$ are the number of pairs in the mixed events and same event, respectively. A onedimentional (1D) correlation function $C(\Delta \varphi)$ is obtained by integrating $C(\Delta \eta, \Delta \varphi)$ over the pseudorapidity range $\Delta \eta$. Another definition of the correlation function is used by the CMS Collaboration [22]:

$$
\frac{1}{N^{\operatorname{tr}}} \frac{d^{2} N^{\text {pair }}}{d \Delta \eta d \Delta \varphi}=B(0,0) \times \frac{S(\Delta \eta, \Delta \varphi)}{B(\Delta \eta, \Delta \varphi)},
$$

where $N^{t r}$ is the number of trigger particles, and the signal and background are:

$$
S(\Delta \eta, \Delta \varphi)=\frac{1}{N^{\operatorname{tr}}} \frac{d^{2} N^{\text {same }}}{d \Delta \eta d \Delta \varphi}, \quad B(\Delta \eta, \Delta \varphi)=\frac{1}{N^{\operatorname{tr}}} \frac{d^{2} N^{\text {mixed }}}{d \Delta \eta d \Delta \varphi} .
$$

This definition depends on event multiplicity, since it involves the number of associated particles where the pair of particles comes with approximately the same $\eta$ and $\varphi$ angles, $B(0,0)$.

The background can be constructed from two singleparticle spectra, $d^{2} N^{\mathrm{tr}} / d \eta d \varphi$ and $d^{2} N^{\mathrm{a}} / d \eta d \varphi$. Instead of correlating every two particles in mixed events, one correlates the yields in the given two bins. The yield represents the average over many events; therefore, the EbE correlations are washed out and the yield of pairs for the background function would be:

$$
B(\Delta \eta, \Delta \varphi)=\int \frac{d^{2} N^{\operatorname{tr}}}{d \eta^{\operatorname{tr}} d \varphi^{\operatorname{tr}}} \frac{d^{2} N^{\mathrm{a}}}{d \eta^{\mathrm{a}} d \varphi^{\mathrm{a}}} \delta_{\mathrm{a}}^{\operatorname{tr}} d \eta^{\mathrm{a}} d \eta^{\operatorname{tr}} d \varphi^{\mathrm{a}} d \varphi^{\operatorname{tr}},
$$

where $\delta_{\mathrm{a}}^{\mathrm{tr}}=\delta\left(\eta^{\mathrm{tr}}-\eta^{\mathrm{a}}-\Delta \eta\right) \delta\left(\varphi^{\mathrm{tr}}-\varphi^{\mathrm{a}}-\Delta \varphi\right)$. Due to the absence of any detector effects in the model, spectra $d N / d \varphi$ as well as a background function $B(\Delta \varphi)$ should be flat. Thus, for function $B(\Delta \varphi, \Delta \eta)$ we use only $d N / d \eta$ distribution and assume flat distribution over $\Delta \varphi$. 
Fourier harmonics $V_{n}$ from Eq. (2) are defined directly from the correlation function $C(\Delta \varphi)$ :

$$
V_{n}=\langle\cos (\Delta \varphi)\rangle=\frac{\sum_{i} C_{i}\left(\Delta \varphi_{i}\right) \cdot \cos \left(n \Delta \varphi_{i}\right)}{\sum_{i} C_{i}\left(\Delta \varphi_{i}\right)} .
$$

If the collective azimuthal anisotropy is the dominant mechanism of the correlation at large $|\Delta \eta|$, then $V_{n}$ coefficients would depend on single-particle anisotropies $v_{n}$ similar to Eq. (3):

$$
V_{n}\left(p_{\mathrm{T}}^{\text {low }}, p_{\mathrm{T}}^{\text {low }}\right)=v_{n}\left(p_{\mathrm{T}}^{\text {low }}\right) \times v_{n}\left(p_{\mathrm{T}}^{\text {low }}\right)+\delta_{n} .
$$

At low $p_{T}$ region the non-flow contribution $\delta_{n}$ is negligible, thus leading to factorization of $V_{n}$. In experiment one usually defines the single-particle flow $v_{n}\{2 P C\}$ via the two-particle correlation (2PC) function using $v_{n}$ at low $p_{\mathrm{T}}$ as a reference,

$$
v_{n}\{2 \mathrm{PC}\}\left(p_{\mathrm{T}}\right)=\frac{V_{n}\left(p_{\mathrm{T}}, p_{\mathrm{T}}^{\text {low }}\right)}{v_{n}\left(p_{\mathrm{T}}^{\text {low }}\right)},
$$

which effectively corresponds to two-particle cumulant method.

Angular dihadron correlations contain all possible types of two-particle correlations. Many sources of twoor many-particle correlations, such as femtoscopic correlations, resonance decays, jets, and collective flow, are presented in the model. The long-range correlations over $\eta$ arise in the model merely due to collective flow. The correlation function $C(\Delta \eta, \Delta \varphi)$ calculated in HYD$\mathrm{JET}++$ in the $\mathrm{Pb}+\mathrm{Pb}$ collision at $\sqrt{s_{\mathrm{NN}}}=2.76 \mathrm{TeV}$ for $2<p_{\mathrm{T}}^{\mathrm{tr}}<4 \mathrm{GeV} / c$ and $1<p_{\mathrm{T}}^{\mathrm{a}}<2 \mathrm{GeV} / c$ is presented in Fig. 1 for the cases of (a) absence of collective flow at zero impact parameter, (b) centrality $0-5 \%$, only elliptic flow $v_{2}$ is turned on, and (c) centrality 0-5\%, both elliptic and triangular flow are present. The generated statistics are about $10^{4}, 10^{4}$, and $10^{5}$ events, respectively. Figure 1(a) shows that the jet peak is highly suppressed at the away-side $\Delta \varphi \approx \pi$ due to jet quenching. Although remnants of it can be seen over a broad $\Delta \eta$ range at the away-side, no long-range azimuthal correlations are seen at the near-side. The long-range azimuthal correlations start to appear at the near-side in the presence of elliptic flow with the characteristic $\cos (2 \Delta \varphi)$ pattern. They are flat in relative pseudorapidity up to $\Delta \eta \approx 4$, which corresponds to a flat pseudorapidity shape of the collective flow in the model. Finally, triangular flow enhances these near-side correlations, often referred to as a ridge. It also modifies the away-side of the distribution by producing a double-hump structure distinctly seen in Fig. 1(c).

In the HYDJET ++ model, $v_{2}$ and $v_{3}$ anisotropies are introduced at the stage of thermal freeze-out, by means of the space modulation of the freeze-out volume and additional modulation of the flow velocity profile for the elliptic flow only. Thus, the model is insensitive to different origins of anisotropy and to the evolution dynamics from the initial state to the freeze-out stage. It is tuned, however, to describe the coefficients $v_{2}$ and $v_{3}$ both at low and at intermediate transverse momenta, where the hadrons from fragmenting jets start to dominate the particle spectrum. The interplay between $v_{2}$ and $v_{3}$ in the final state leads to the appearance of higher order flow harmonics, which reasonably describe data at mid-central collisions 20.

The results for long-range azimuthal correlations obtained with Eq. (5) for $1<p_{\mathrm{T}}^{\mathrm{a}}<1.5 \mathrm{GeV} / c$ and $3<p_{\mathrm{T}}^{\text {tr }}<3.5 \mathrm{GeV} / c$ in HYDJET ++ calculations are plotted in Fig. 2 onto the CMS data 22 for different centralities. Since the correlation function given by Eq. (5) depends on the multiplicity of associated particles, it does not always exactly coincide with the model. Therefore, HYDJET + + calculations are shifted on the constant value in such a way that the minima of $C(\Delta \varphi)$ in the data and in the model coincide. In central collisions the model underestimates the data a bit while in peripheral collisions the tendency is the opposite. The semi-central collisions are described quite well. Note that for centralities up to $35 \%$ the difference between peak magnitudes of the HYDJET ++ distributions and the experimental ones is less than $3 \%$. It increases to $12 \%$ in peripheral collisions with centrality $50-60 \%$. To see the role of each of the Fourier coefficients $V_{n}$ more distinctly, we plot in Fig. 3 the values of the first five $V_{n}$ coefficients, calculated for the distributions shown in Fig. 2. At very central collisions all coefficients $V_{n}$ in the model are lower than those extracted from the data. At semi-central, semi-peripheral and even peripheral collisions all but $V_{1}$ and $V_{2}$ describe data rather well. At peripheral collisions $V_{2}$ in the model is higher than in data. This circumstance reflects the fact that the model predicts higher single-particle elliptic flow in the region of intermediate transverse momenta $3<p_{\mathrm{T}}<3.5 \mathrm{GeV} / c$ compared to the data, see [20, while the factorization holds. Note that there is no directed flow $v_{1}$ in the model, neither pseudorapidity odd $v_{1}$ nor even $v_{1}$, which is supposed to come from the initial state fluctuations as discussed in the literature 23. Nevertheless, the $V_{1}$ component appears here due to violation of the momentum conservation, because in a part of the system with selected $p_{T}$ and $\eta$ ranges the momentum is not conserved. It was shown in 24 that the contribution of momentum conservation to the $V_{1}$ component can be presented by the term

$$
V_{1 m . c .}=-\frac{p_{\mathrm{T}}^{\mathrm{tr}} p_{\mathrm{T}}^{\mathrm{a}}}{M\left\langle p_{\mathrm{T}}^{2}\right\rangle}
$$

where $M$ and $\left\langle p_{\mathrm{T}}^{2}\right\rangle$ are the multiplicity and average squared transverse momentum of the whole event, respectively. This approximation was made under assumption that the transverse momentum distribution is isotropic, or anisotropy is very weak and can be neglected. At higher $p_{\mathrm{T}}$ the cut on $\Delta \eta$ introduces additional unbalance in $\Delta \varphi$-distribution, since the near-side jet peak is almost completely eliminated by the cut, whereas the away-side jet peak stays partially. Figure 4 displays the $V_{1}\left(p_{\mathrm{T}}^{\text {tr }}\right)$ component calculated in HYDJET ++ for different momenta of associated particles at two selected central- 

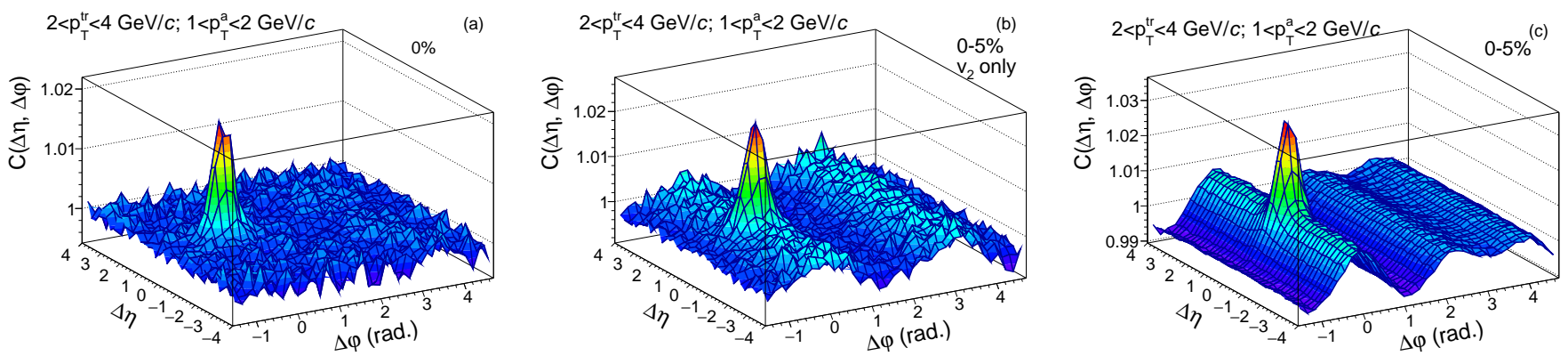

FIG. 1. (Color online) Two-dimensional correlation function in HYDJET ++ in $\mathrm{Pb}+\mathrm{Pb}$ collisions at $\sqrt{s_{\mathrm{NN}}}=2.76$ TeV for $2<p_{\mathrm{T}}^{\text {tr }}<4 \mathrm{GeV} / c$ and $1<p_{\mathrm{T}}^{\mathrm{a}}<2 \mathrm{GeV} / c$ for (a) central collisions with impact parameter $b=0 \mathrm{fm}$, no flow, (b) centrality $0-5 \%$ with only elliptic flow, and (c) centrality $0-5 \%$ with both elliptic and triangular flow present.

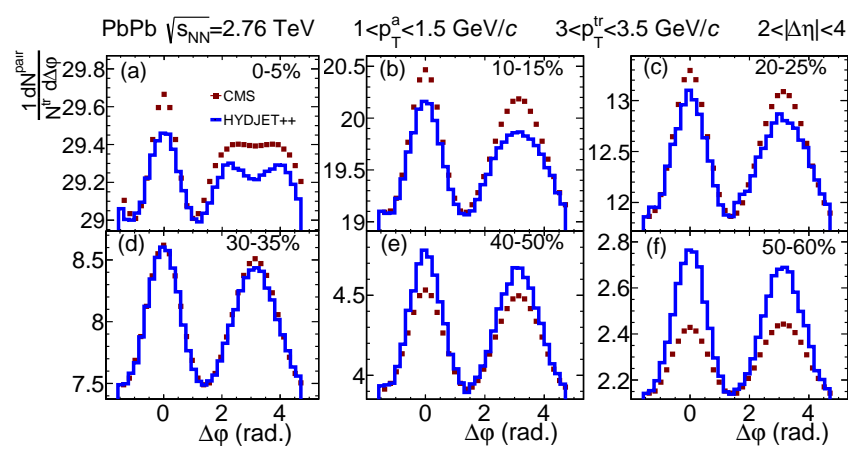

FIG. 2. (Color online) One-dimensional correlation function at $2<|\Delta \eta|<4$ in HYDJET ++ in $\mathrm{Pb}+\mathrm{Pb}$ collisions at $\sqrt{s_{\mathrm{NN}}}=$ $2.76 \mathrm{TeV}$ for $3<p_{\mathrm{T}}^{\mathrm{tr}}<3.5 \mathrm{GeV} / c$ and $1<p_{\mathrm{T}}^{\mathrm{a}}<1.5 \mathrm{GeV} / c$ for different centralities in comparison with CMS data [22].

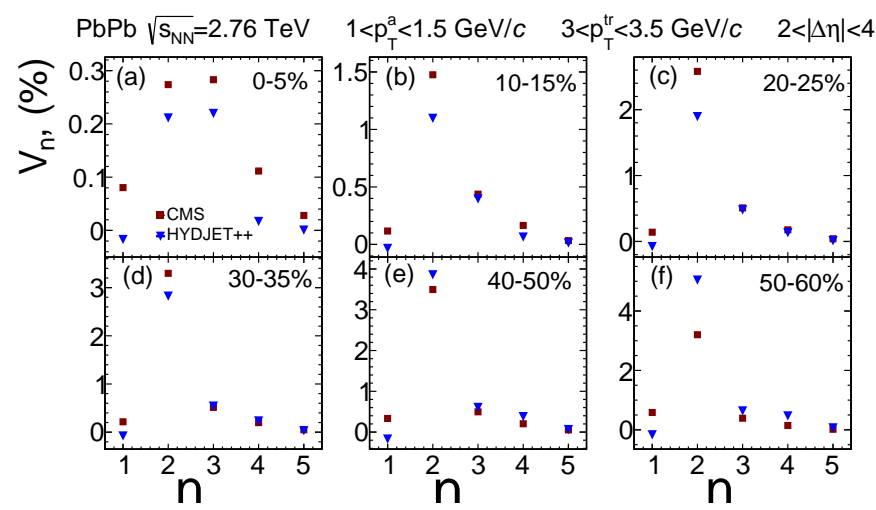

FIG. 3. (Color online) Fourier coefficients $V_{n}$ extracted for the $1 \mathrm{D}$ correlation function, presented in Fig. 2, in comparison with CMS data 22].

ities. Available experimental data of ALICE and CMS collaborations are plotted onto the model results as well. It is clearly seen that the distributions can be approximated by a linear function only at a low- $p_{T}$ interval. Thus, the estimation of the $V_{1 . m . c}$ contribution to the $V_{1}$ measured in the whole transverse momentum range requires additional study. Figure 5 shows the coefficients

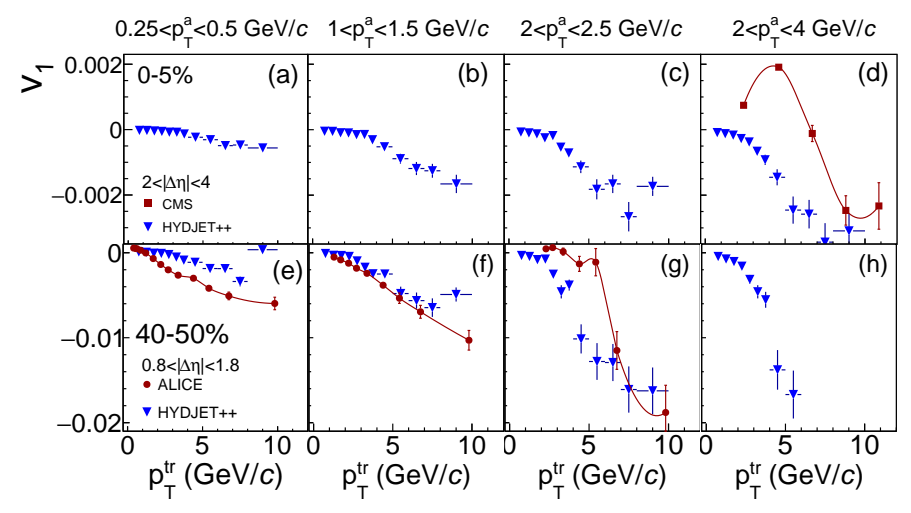

FIG. 4. (Color online) Upper row: Coefficients $V_{1}$ in the 0$5 \%$ central $\mathrm{Pb}+\mathrm{Pb}$ collision at $\sqrt{s_{\mathrm{NN}}}=2.76 \mathrm{TeV}$ for different associated $p_{\mathrm{T}}^{\mathrm{a}}$ as a function of $p_{\mathrm{T}}^{\mathrm{tr}}$ for HYDJET ++ (circles) and CMS (squares) data 25. Bottom row: The same as the top, but for centrality $40-50 \%$. Squares represent ALICE data from [14. Lines are drawn to guide the eye.

$v_{2}\{2 P C\}, v_{3}\{2 P C\}$, and $v_{4}\{2 P C\}$ extracted from $V_{n}$ at $|\Delta \eta|>2$ by Eq. (7) with $p_{\mathrm{T}}^{\mathrm{tr}}=p_{\mathrm{T}}^{\mathrm{a}}$ in comparison with $v_{2}, v_{3}, v_{4}$ calculated with respect to the known reaction plane at the generator level. In case of negative $V_{n}$ the coefficients $v_{n}\{2 P C\}$ are taken as $v_{n}\{2 \mathrm{PC}\}=-\sqrt{\left|V_{n}\right|}$. Comparison is presented for two centralities, $0-5 \%$ and $30-35 \%$. One can see that in the range of $p_{\mathrm{T}}<3.5 \mathrm{GeV} / c$ the $2 \mathrm{PCmethod}$ describes the $v_{n}$ coefficients pretty well. This means that $V_{n}$ coefficients factorize in this region into a product of two singular flow coefficients, and the collective flow is the dominant source of correlation. At higher transverse momenta, $p_{\mathrm{T}}>3.5 \mathrm{GeV} / c$, the nonflow contribution of the jet component dominates. This contribution is negative for odd coefficients $v_{n}\{2 \mathrm{PC}\}$.

It is worth noting that higher order coefficients $V_{n}$, $n>4$ also appear in the model in $C(\Delta \varphi)$ decomposition, though they decrease rapidly with $n$, as shown in Fig. 3 . These coefficients at low $p_{T}$ can only originate from the lower order flow harmonics, $v_{2}$ and $v_{3}$. Figure 6 depicts pentagonal flow, $v_{5}\{2 P C\}$, obtained by Eq. (7) at different centralities. The result is compared to the product 


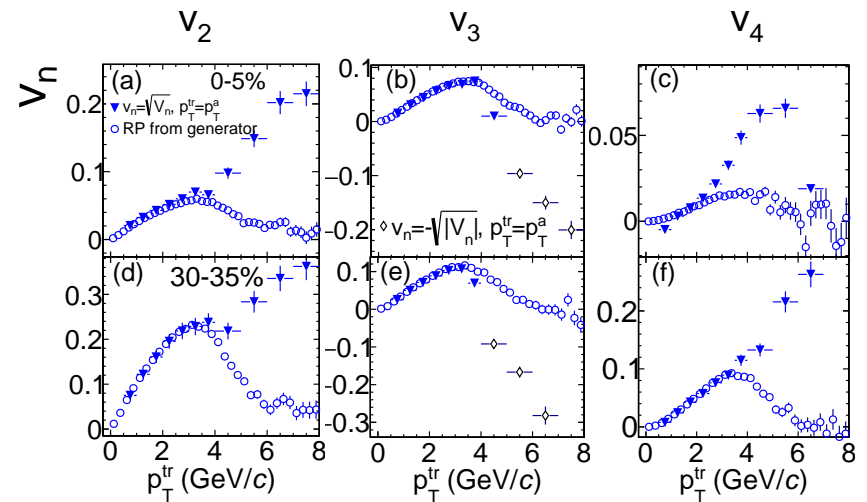

FIG. 5. (Color online) Upper row: Single-particle coefficients $v_{n}$ obtained with respect to a known reaction plane (open circles) and $v_{n}\{2 \mathrm{PC}\}$ extracted from $V_{n}$ (full triangles down) for the same $p_{\mathrm{T}}^{\mathrm{a}}=p_{\mathrm{T}}^{\mathrm{tr}}$ in HYDJET ++ generated $0-5 \%$ central $\mathrm{Pb}+\mathrm{Pb}$ collisions at $\sqrt{s_{\mathrm{NN}}}=2.76 \mathrm{TeV}$. Open diamonds indicate points with negative $V_{n}$. Bottom row: The same as the top, but for $30-35 \%$ centrality.

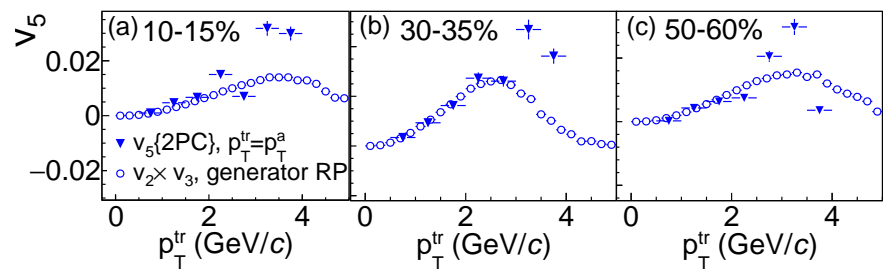

FIG. 6. (Color online) $v_{5}\{2 \mathrm{PC}\}$ (triangles) obtained by Eq. (7) at centrality 10-15\% (left plot), 30-35\% (middle plot), and $50-60 \%$ (right plot) in comparison to the product $v_{2}\left(p_{T}\right) \times v_{3}\left(p_{\mathrm{T}}\right)$ (circles), obtained at generator levels with known reaction planes.

$v_{2}\left(p_{T}\right) \times v_{3}\left(p_{T}\right)$, obtained at the generator level with a known reaction plane. It shows that the substantial contribution to $V_{5}$ comes from $v_{2}$ and $v_{3}$ harmonics at all centralities at $p_{T} \geq 1 \mathrm{GeV} / c$.

\section{CONCLUSIONS}

The HYDJET ++ model allows us to study the influence of a single harmonic, such as $v_{2}$ or $v_{3}$, as well as theirinterplay, on the final particle azimuthal distributions. This is the ideal situation, where all genuine higher-order initial fluctuations, which can distort the signal, are simply switched off. Elliptic flow contributes to all even harmonics of higher order, whereas the interplay of $v_{2}$ and $v_{3}$ leads to the appearance of odd harmonics in the model. In the present paper we obtained clear evidence that this mechanism allows one to describe also the dihadron correlations, including the double-hump structure, at midcentral collisions, where lower orders of collective flow dominates over higher harmonics. The measured amplitude of the ridge at mid-central collisions is well described by a superposition of elliptic and triangular flows. This is the main result of the paper.

Also, for pairs of particles with a large pseudorapidity gap $(|\Delta \eta|>2)$ in a range of transverse momenta $p_{\mathrm{T}}<3.5$ $\mathrm{GeV} / c$, the coefficients $V_{2}, V_{3}$, and $V_{4}$ are found to factorize into the product of corresponding collective flow coefficients $v_{n}$ calculated in the model with a known reaction plane. In the absence of initial pentagonal fluctuations, pentagonal coefficient, $v_{5}\{2 P C\}$, extracted from the dihadron correlation function follows approximately the scaling condition $v_{5}\{2 \mathrm{PC}\} \propto v_{2} v_{3}$ at $p_{T} \leq 2.5 \mathrm{GeV} / c$ only.

\section{ACKNOWLEDGMENTS}

We are grateful to L. V. Malinina for the fruitful discussions and valuable comments. This publication was supported by the Russian Scientific Fund under Grant No. 14-12-00110 in a part of computer simulation of 2D correlation functions in $\mathrm{Pb}+\mathrm{Pb}$ collisions and extraction of the flow Fourier coefficients $V_{\mathrm{n}}$. G.E. acknowledges the European Social Fund within the framework of realizing the project Support of Inter-sectoral Mobility and Quality Enhancement of Research Teams at Czech Technical University in Prague, CZ.1.07/2.3.00/30.0034.
[1] A. Adare et al. (PHENIX Collaboration), Phys. Rev. C 78, 014901 (2008).

[2] M.M. Aggarwal et al. (STAR Collaboration), Phys. Rev. C 82, 024912 (2010).

[3] K. Aamodt et al. (ALICE Collaboration), Phys. Rev. Lett. 107, 032301 (2011).

[4] H. Stoecker, Nucl. Phys. A750, 121 (2005).

[5] J. Casalderrey-Solana, E. Shuryak, and D. Teaney, J. Phys. Conf. Ser. 27, 22 (2005).

[6] I.M. Dremin, Nucl. Phys. A767, 233 (2006).

[7] J. Alam, S. Chattopadhyay, T. Nayak, B. Sinha, and Y. P. Viyogi (eds.), in Proceedings of Quark Matter 2008 (IOP Publishing, Bristol, 2008) [J. Phys. G: Nucl. Part.
Phys. 35(10) (2008)].

[8] B. Alver and G. Roland, Phys. Rev. C 81, 054905 (2010) [Erratum-ibid. C 82, 039903 (2010)].

[9] S. Chatrchyan et al. (CMS Collaboration), Phys. Lett. B 718, 795 (2013).

[10] G. Aad et al. (ATLAS Collaboration), Phys. Rev. Lett. 110, 182302 (2013).

[11] B. Abelev et al. (ALICE Collaboration), Phys. Lett. B 719, 29 (2013).

[12] S. Chatrchyan et al. (CMS Collaboration), Phys. Lett. B 724, 213 (2013).

[13] V. Khachatryan et al. (CMS Collaboration), JHEP 1009, 091 (2010). 
[14] K. Aamodt et al. (ALICE Collaboration), Phys. Let. B 708, 249 (2012).

[15] G. Aad et al. (ATLAS Collaboration), Phys. Rev. C 86, 014907 (2012).

[16] I.P. Lokhtin, L.V. Malinina, S.V. Petrushanko, A.M. Snigirev, I. Arsene, and K. Tywoniuk, Comput. Phys. Commun. 180, 779 (2009).

[17] T. Sjostrand, S. Mrenna, and P. Skands, JHEP 0605 , 026 (2006).

[18] I.P. Lokhtin and A.M. Snigirev, Eur. Phys. J. C45, 211 (2006).

[19] N.S. Amelin, R. Lednicky, I.P. Lokhtin, L.V. Malinina, A.M. Snigirev, Iu.A. Karpenko, Yu.M. Sinyukov, I. Arsene, and L. Bravina, Phys. Rev. C 77, 014903 (2008).

[20] L.V. Bravina, B.H. Brusheim Johansson,
G.K. Eyyubova, V.L. Korotkikh, I.P. Lokhtin, L.V. Malinina, S.V. Petrushanko, A.M. Snigirev, and E.E. Zabrodin, Eur. Phys. J. C74, 2807 (2014).

[21] L.V. Bravina, B.H. Brusheim Johansson, E.E. Zabrodin, G.K. Eyyubova, V.L. Korotkikh, I.P. Lokhtin, L.V. Malinina, S.V. Petrushanko, and A.M. Snigirev, Phys. Rev. C 89, 024909 (2014).

[22] S. Chatrchyan et al. (CMS Collaboration), Eur. Phys. J. C72, 2012 (2012).

[23] D. Teaney and L. Yan, Phys. Rev. C 83, 064904 (2011).

[24] N. Borghini, P.M. Dinh, and J.-Y. Ollitrault, Phys. Rev. C 62, 034902 (2000).

[25] S. Chatrchyan et al. (CMS Collaboration), JHEP 1107, 076 (2011). 